\title{
Comunicação terapêutica entre enfermeiros e pacientes de uma unidade hospitalar
}

\author{
Therapeutic communication between nurses and patients from a hospital unit
}

\author{
Comunicación terapéutica entre enfermeras y pacientes del hospital
}

Patrícia de Lemos Negreiros', Mayara de Oliveira Fernandes", Kátia Nêyla de Freitas Macedo-Costa"', Grazielle Roberta Freitas da Silvalv

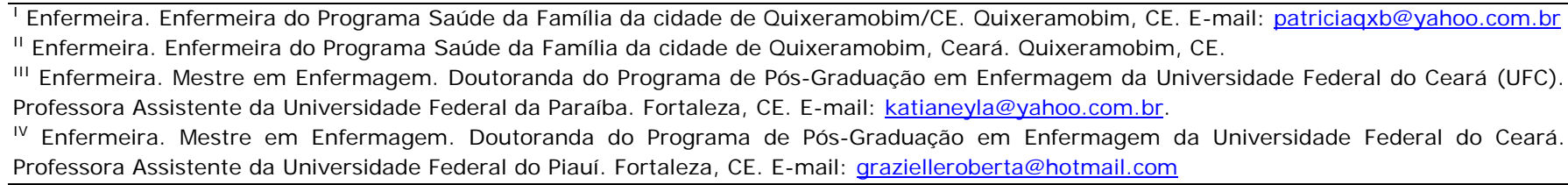

\section{RESUMO}

A comunicação é uma competência diária dos enfermeiros, sendo fator determinante na relação de ajuda e indicador na avaliação dos cuidados prestados. Dessa forma, objetivou-se descrever a comunicação terapêutica entre enfermeiros e pacientes no ambiente hospitalar. Trata-se de estudo descritivo-exploratório, com abordagem quantitativa, realizado em um hospital do estado do Ceará em outubro/2007, com cinco enfermeiros, mediante observação sistemática das interações estabelecidas com os pacientes internados. Para registro dos dados utilizou-se check-list, contendo estratégias para efetivação da comunicação, segundo cada interação. Das 21 interações, algumas se direcionam exclusivamente à prática comunicativa, como as orientações aos pacientes. Outras se referiram aos seguintes procedimentos: sondagem vesical; verificação de sinais vitais; realização de curativos; acompanhamento da evolução e trabalho de parto; punção venosa e vacinação. Do total de interações, o enfermeiro $C$ foi o profissional que mais se comunicou de modo terapêutico (42,9\%). Das demais 12 interações $(57,1 \%)$, realizadas pelos enfermeiros A, B, D e E, oito foram consideradas não-terapêuticas. Dessas, o item mais utilizado foi o Uso de jargão profissional, importante fator que pode interferir negativamente no prognóstico do paciente. Os enfermeiros precisam conhecer e implementar no seu cotidiano as estratégias de comunicação terapêutica como forma de atender as necessidades dos pacientes.

Descritores: Comunicação; Enfermagem; Cuidados de Enfermagem.

\section{ABSTRACT}

Communication is an everyday competence of nurses, factor in the aid relationship and indicator of the care evaluation. This work aimed to describe the therapeutic communication between nurses and patients in the hospital. It is a descriptive exploratory study with a quantitative approach, conducted in a hospital from the state of Ceará in October of 2007, with 05 nurses, through systematic observation of interactions established with the inpatients. A check-list was used to record the data, containing strategies for effective communication, in each interaction. From the 21 interactions, some are directed exclusively to communicative practice, such as the guidelines to patients. Others referred to the following procedures: vesical catheterization; checking vital signs, wound dressing, monitoring progress and labor; venipuncture and immunization. From the interactions, the nurse $C$ was the professional who communicated in the most therapeutic way (42.9\%). From the remaining 12 interactions (57.1\%), performed by nurses A, B, D and E, 8 were non-therapeutic. Out of these, the most used item was the professional jargon, an important factor that can negatively affect patient outcomes. Nurses must know and implement in their routine strategies for therapeutic communication as a way to meet the needs of patients.

Descriptors: Communication. Nursing, Nursing Care.

\section{RESUMEN}

La comunicación es una habilidad diaria de los enfermeros, siendo el factor determinante en la relación de ayuda y indicador en la evaluación de las atenciones ofrecidas. Así, se objetivó describir la comunicación terapéutica entre los enfermeros y pacientes en el ambiente hospitalario. Se trata de un estudio descriptivo y exploratorio, con abordaje cuantitativo, realizado en un hospital del estado del Ceará en outubro/2007, con 05 enfermeros, mediante observación sistemática de las interacciones establecidas con los pacientes internados. Para registro de los datos, se utilizó el check-list, conteniendo estrategias para efetivación de la comunicación, según cada interacción. De las 21 interacciones, algunas se direccionan exclusivamente a la práctica comunicativa, como las orientaciones a los pacientes. Otras se referieron a los seguintes procedimientos: sondaje vesical; verificación de señales vitales; realización de curativos; acompañamiento de la evolución y trabajo de parto; punción venosa y vacunación. Del total de interacciones, el enfermero $C$ fue el profesional que más se comunicó de modo terapéutico (42,9\%). De las 12 interacciones restantes $(57,1 \%)$, realizadas por los enfermeros A, B, D y E, 8 fueron consideradas no terapéuticas. De estos, el ítem más utilizado fue el Uso de jerga profesional, importante factor que puede interferir negativamente en el pronóstico del paciente. Los enfermeros necesitan conocer y implementar en su rutina las estrategias de comunicación terapéutica como forma de atender a las necesidades de los pacientes.

Descriptores: Comunicación; Enfermería; Atención de Enfermería. 


\section{NTRODUÇÃO}

A comunicação é uma ação de competência diária dos profissionais de saúde, sendo determinante na relação de ajuda e indicador na avaliação dos cuidados prestados, principalmente na enfermagem ${ }^{(1)}$. Essa competência é necessária em todas as ações de enfermagem realizadas, influenciando diretamente a interação com o paciente. Durante o processo de hospitalização, é fundamental proporcionar um novo modo de olhar e agir dos profissionais em relação aos pacientes que estão sob seus cuidados. Comprovadamente existe ineficácia da comunicação da equipe de enfermagem no âmbito hospitalar ${ }^{(1-2)}$

Na hospitalização o paciente permanece fora do seu ambiente familiar e é exposto a um ambiente completamente estranho, onde rotinas e normas controlam e determinam suas ações, aspecto esse, que nem sempre é considerado pelos profissionais que $o$ atendem. Os instrumentos básicos da relação de ajuda, tais como o diálogo e a empatia podem ajudar a diminuir os problemas apresentados pela pessoa hospitalizada, mas como não são trabalhados, tem como consequência falhas na comunicação(3).

Desse modo, os profissionais de enfermagem devem utilizar a comunicação como instrumento para humanizar o cuidado, dialogando com o paciente para esclarecer dúvidas quanto ao seu tratamento, exames diagnósticos ou procedimentos clínicos, minimizando sua ansiedade causada pela sua condição de passividade imposta pela doença e hospitalização(2).

A interação entre o cuidador e o ser cuidado se apresenta como possibilidade de construção de práticas assistenciais humanizadas ${ }^{(2)}$, logo, é inegável a relevância de estudos que se ocupem dessa problemática. Nesse contexto, a comunicação terapêutica se constitui uma mola impulsionadora no concernente à humanização do cuidado em enfermagem.

A comunicação terapêutica consiste na habilidade do profissional em usar seu conhecimento sobre comunicação, para ajudar a pessoa com tensão temporária, a conviver com outras pessoas e ajustarse ao que não pode ser mudado e a superar os bloqueios à auto-realização para enfrentar seus problemas. Com base na revisão de literatura, em pesquisas, e na sua própria experiência de observação diária, Stefanelli ${ }^{(4)}$, classificou as técnicas de comunicação terapêutica em três grupos: Expressão, Clarificação e Validação.

No grupo Expressão estão arroladas as técnicas que ajudam à descrição da experiência e a expressão de pensamentos e sentimentos sobre essa. As técnicas de expressão são as mais utilizadas no início do relacionamento terapêutico enfermeiro-paciente. As técnicas são as seguintes ${ }^{(4)}$ : Usar Terapeuticamente o Silêncio; Ouvir Reflexivamente;
Verbalizar Aceitação; Verbalizar Interesse; Usar Frases Incompletas; Repetir Comentários Feitos Pelo Paciente; Repetir as Últimas Palavras ditas pelo Paciente; Fazer Pergunta; Usar Frases Descritivas; Manter o Paciente no Mesmo Assunto; Permitir ao Paciente que Escolha o Assunto; Colocar em Foco a Idéia Principal; Verbalizar Dúvidas; Dizer Não; Estimular Expressão de Sentimentos Subjacentes e Usar Terapêutico do Humor.

Já no grupo de Clarificação são apresentadas técnicas que ajudam o profissional a esclarecer o que for expresso pelo paciente. São elas: Estimular Comparações; Solicitar ao Paciente que Esclareça Termos Comuns e Solicitar ao Paciente Que Precise o Agente de Ação. E por fim, o grupo de Validação, no qual são introduzidas as técnicas que permitem a existência de significação comum do que é expresso.

A validação da comunicação deve acompanhar todo processo de relacionamento terapêutico e é necessária porque mensagens emitidas devem ter a mesma significação para as pessoas envolvidas no processo terapêutico. Nesse grupo se encontram as seguintes técnicas: Repetir a Mensagem do Paciente; Pedir ao Paciente para Repetir o que foi dito e Sumarizar o Conteúdo da Interação. Além desses grupos são recomendadas também condutas que devem ser evitadas para não se chegar à comunicação não-terapêutica, que não se constitui foco desse estudo(4).

Para se obter maior percepção acerca do processo comunicativo, não como mero recurso teórico, mas com finalidades práticas, a comunicação efetiva e eficaz diária pode ajudar na conduta dos enfermeiros e assegurar possibilidades terapêuticas na sua atuação, bem como, desempenho humanista e direcionado ao bem estar daqueles que estão sob seus cuidados ${ }^{(3)}$

Diante do exposto, teve-se como objetivo no presente estudo descrever e analisar o processo de comunicação terapêutica entre enfermeiros e pacientes no ambiente hospitalar, à luz de Stefanelli ${ }^{(4)}$.

\section{METODOLOGI A}

Estudo descritivo, exploratório e observacional, com abordagem quantitativa, desenvolvido em um hospital de um município do estado do Ceará. A instituição foi escolhida, inicialmente, por ser referência para a região a qual se localiza, onde oferece serviços de clínica médica, clínica cirúrgica, obstetrícia, emergência e traumatologia.

Foram selecionados os setores de clínica médica, cirúrgica e obstetrícia, pois nesses o período de internação é de no mínimo dois dias, assim o contato entre enfermeiros e pacientes apresenta maiores possibilidades na utilização das técnicas comunicativas. As unidades de emergência e traumatologia são setores dinâmicos e com alta 
rotatividade, impossibilitando a interação mais contínua.

A clínica cirúrgica possui 12 leitos e o setor obstétrico 16 leitos, sendo que 08 estão na sala de pré-parto e parto e 08 no alojamento conjunto. Já a clínica médica, 09 enfermarias com 32 leitos. A instituição conta com 14 enfermeiros, dos quais, todos atuam nas três clínicas especificadas.

O estudo desenvolveu-se no mês de Outubro de 2007 durante um período de 4 horas/dia em média, nos três turnos de acordo a escala de cada profissional a ser observado. Os sujeitos que aceitaram fazer parte do estudo foram cinco enfermeiros, que tinham disponibilidade no período de coleta. Do total de 14 enfermeiros, três não aceitaram e os demais não trabalharam no mês de julho, período de coleta.

Optou-se pela técnica de observação sistemática não participante, caracterizada pelo planejamento prévio e a utilização de anotações, recorrendo ao uso de recursos técnicos, mecânicos ou eletrônicos ${ }^{(5)}$. O observador deliberadamente se mantém na posição de observador e espectador, evitando se envolver ou deixar-se envolver com o objeto da observação. Para tanto, utilizou-se dois instrumentos, um check-list adaptado por Stefanelli ${ }^{(4)}$, e o segundo contemplou os dados profissionais e de identificação.

Para a condução do estudo foram contempladas todas as normas da Resolução 196/96 do Conselho Nacional de Saúde, no qual foi apreciado e aprovado pelo Comitê de Ética em Pesquisa do Complexo Hospitalar da Universidade Federal do Ceará, sob protocolo 243/07. Os objetivos da pesquisa foram apresentados aos enfermeiros que se disponibilizaram a participar do estudo e após assinaram o Termo de Consentimento Livre e Esclarecido. Dado o início da coleta, os profissionais não sabiam quando e nem qual procedimento exatamente estava sendo avaliado, sendo que estes foram identificados com as letras A, B, C, D e E.

Vale esclarecer que a pesquisadora adentrou ao campo de pesquisa 07 dias antes da coleta, para fazer parte da realidade observada. Esses cuidados foram tomados para evitar mudanças drásticas no comportamento dos profissionais.

Os dados gerais da observação foram agrupados em duas tabelas, as quais contemplaram os profissionais, as interações realizadas e os itens pertinentes à comunicação terapêutica. Achou-se conveniente descrever cada interação observada, para analisar com maior profundidade a comunicação observada. A análise se deu à luz do referencial teórico e da literatura pertinente, para comparações.

\section{RESULTADOS E DISCUSSÃO}

Dos cinco enfermeiros que participaram do estudo, três são do sexo feminino e dois do masculino, a faixa etária variou de 24 a 56 anos e o período de exercício da profissão oscilou de 01 a 22 anos.

Dos profissionais, dois trabalham integralmente na assistência hospitalar e três atuam não somente no hospital, mas também na Estratégia Saúde da Família. Na Tabela 1, as interações ocorridas entre enfermeiros e pacientes de acordo com cada profissional observado. 
Tabela 1: Distribuição dos enfermeiros de acordo com as interações estabelecidas durante a assistência de enfermagem. Quixeramobim, Ceará, 2007.

\begin{tabular}{|c|c|c|c|}
\hline Enfermeiros & I nterações & Total de interações & $\%$ \\
\hline A & $\begin{array}{l}\text { Visita aos Pacientes } \\
\text { Sondagem Vesical } \\
\text { Orientações ao paciente sobre cirurgia } \\
\text { Verificação da P.A } \\
\text { Avaliação das parturientes }\end{array}$ & 5 & 23,8 \\
\hline B & $\begin{array}{l}\text { Visita aos pacientes } \\
\text { Realização de curativo cirúrgico I } \\
\text { Realização de curativo cirúrgico II }\end{array}$ & 3 & 14,3 \\
\hline $\mathrm{C}$ & $\begin{array}{l}\text { Visita aos pacientes } \\
\text { Orientações ao paciente sobre cirurgia } \\
\text { Verificação da P.A e temperatura } \\
\text { Toque vaginal } \\
\text { Acompanhamento da evolução e do trabalho de parto vaginal } \\
\text { Orientações à parturiente } \\
\text { Orientações às puérperas } \\
\text { Orientações a paciente que se submeteria à cesariana } \\
\text { Punção venosa }\end{array}$ & 9 & 42,9 \\
\hline $\mathrm{D}$ & Acompanhamento em punção de subclávia & 1 & 4,7 \\
\hline $\mathrm{E}$ & $\begin{array}{l}\text { Vacinação das puérperas } \\
\text { Sondagem vesical } \\
\text { Realização de curativo contaminado }\end{array}$ & 3 & 14,3 \\
\hline Total & - & 21 & 100 \\
\hline
\end{tabular}

Foram observadas 21 interações, das quais algumas se direcionam exclusivamente à prática comunicativa, como é o caso das orientações. Em contrapartida, outras interações requereram além da comunicação, a realização dos procedimentos, a saber: sondagem vesical, verificação de sinais vitais, realização de curativos, acompanhamento da evolução e trabalho de parto, punção venosa e vacinação.

Dos profissionais observados, o enfermeiro $C$ foi o que mais estabeleceu contato com os pacientes, 09 $(42,9 \%)$ das 21 interações, dentre elas, citam-se: visita aos pacientes, orientações pré-operatórias, verificação da P.A e temperatura, toque vaginal, acompanhamento da evolução e trabalho de parto normal, orientações à parturiente, orientações às puérperas e à paciente que se submeteria à cesariana e punção venosa.

Já o segundo profissional que mais promoveu interações foi o enfermeiro A, 05 (23,8\%), sendo citadas: visita aos pacientes, sondagem vesical, orientações ao paciente sobre cirurgia, verificação da P.A e avaliação das parturientes. Os enfermeiros B e E realizaram três $(14,3 \%)$ interações cada. O enfermeiro $B$ executou: visita aos pacientes, realização de dois curativos. Já o Enfermeiro E, fez: vacinação das puérperas, sondagem vesical e realização de curativo contaminado. Por último o enfermeiro D estabeleceu interação durante acompanhamento de punção de subclávia.
No referente aos grupos da comunicação terapêutica, 16 itens do grupo de EXPRESSÃO foram utilizados, como mostra a Tabela 2 abaixo. 
Tabela 2: Distribuição dos itens pertinentes ao grupo de EXPRESSÃO, mediante interações realizadas durante a assistência de enfermagem. Quixeramobim. Ceará, 2007

\begin{tabular}{|c|c|c|c|c|c|c|}
\hline \multirow{2}{*}{ I tens relacionados ao grupo de expressão } & \multicolumn{6}{|c|}{ Enfermeiros } \\
\hline & A & B & C & D & $\mathbf{E}$ & Total \\
\hline 1. Permanecer em Silêncio & - & 1 & 7 & 1 & 2 & 11 \\
\hline 2. Ouvir Reflexivamente & - & - & 6 & - & - & 6 \\
\hline 3. Verbalizar Aceitação & - & - & 3 & - & - & 3 \\
\hline 4. Verbalizar Interesse & 2 & - & 9 & - & 1 & 12 \\
\hline 5. Usar frases incompletas & 2 & - & 3 & - & - & 5 \\
\hline 6. Repetir comentários dos pacientes & 1 & - & 2 & - & - & 3 \\
\hline 7. Repetir as últimas palavras do paciente & 3 & - & 3 & - & 1 & 7 \\
\hline 8. Fazer perguntas & 3 & 2 & 7 & - & 2 & 14 \\
\hline 9. Fazer perguntas relativas aos dados comunicados & 2 & 1 & 4 & - & 1 & 8 \\
\hline 10. Introduzir problemas relacionados & - & - & 1 & - & - & 1 \\
\hline 11. Devolver a expressão feita pelo paciente & - & - & 1 & - & - & 1 \\
\hline 12. Usar frases descritivas & - & 1 & 9 & - & 2 & 12 \\
\hline 13. Manter o paciente no mesmo assunto & - & 1 & 2 & - & - & 3 \\
\hline 14. Colocar em foco a idéia principal & 1 & 1 & 4 & - & - & 6 \\
\hline 15. Dizer “Não" & - & - & 1 & - & - & 1 \\
\hline 16. Estimular expressão de sentimentos subjacentes & - & - & 1 & - & - & 1 \\
\hline \begin{tabular}{|c|} 
Total \\
\end{tabular} & 14 & 7 & 63 & 1 & 9 & 94 \\
\hline
\end{tabular}

Ao todo, o enfermeiro A mediante as 05 interações que realizou, comunicou-se terapeuticamente, de acordo com o grupo de expressão por 14 vezes. O enfermeiro B de acordo com as 03 interações que realizou, comunicou-se 07 vezes. O enfermeiro $C$ nas suas 09 interações comunicou-se 63 vezes. O enfermeiro D mediante interação que estabeleceu comunicou-se uma vez e o enfermeiro $E$ nas suas 03 interações, comunicou-se expressivamente por nove vezes.

Os itens que mais se apresentaram nas interações foram os seguintes, em ordem decrescente: 8. FAZER PERGUNTAS (14 vezes); 12. USAR FRASES DESCRITIVAS (12 vezes); 4. VERBALIZAR INTERESSE (11 vezes) e 1. PERMANECER EM SILÊNCIO (11 vezes).

$O$ item FAZER PERGUNTAS foi executado três vezes pelo enfermeiro $A$, duas pelo enfermeiro $B$, sete pelo profissional $C$ e duas pelo enfermeiro $E$. USAR FRASES DESCRITIVAS esteve presente uma vez nas interações do enfermeiro $B$, nove na atuação do enfermeiro $C$, duas vezes praticados pelo profissional E. O item VERBALIZAR INTERESSE, duas vezes pelo enfermeiro $A$, nove pelo enfermeiro $C$ e uma pelo enfermeiro $E$. O item PERMANECER EM SILÊNCIO, mediante as 21 interações mostradas na Tabela 1 , foi utilizado uma vez pelo enfermeiro B, sete pelo enfermeiro $C$, uma vez pelo enfermeiro $D$ e duas vezes pelo enfermeiro $\mathrm{E}$.

Referente ao grupo de CLARIFICAÇÃO, composto por quatro itens segundo Stefanelli ${ }^{(4)}$, apenas dois enfermeiros utilizaram-no quando interagiam com os pacientes. A citar os itens SOLICITAR QUE ESCLAREÇA TERMOS INCOMUNS, utilizado uma vez pelo enfermeiro $C$ e o item DESCREVER OS EVENTOS EM SEQUÊNCIA LÓGICA, também praticado pelo profissional $C$ em cinco das suas interações. Os demais enfermeiros: $A, B, D$ e $E$ não utilizaram nenhum item do grupo de CLARIFICAÇÃO.

Dos três itens pertencentes ao grupo de VALIDAÇÃO, apenas dois foram utilizados pelos enfermeiros observados, a saber: REPETIR A MENSAGEM DO PACIENTE, executado por duas vezes, tanto pelo enfermeiro $A$ quanto pelo $C$ e SUMARIZAR O QUE FOI DITO NA INTERAÇÃO, praticado três vezes pelo enfermeiro $C$. De um modo geral, o enfermeiro $C$ embasou sua comunicação no terceiro grupo da comunicação terapêutica por cinco vezes; o enfermeiro $A$ o fez por duas vezes e os profissionais $B, D$ e $E$ não praticaram o processo comunicativo dentro do grupo de validação.

A seguir será analisada a atuação de cada enfermeiro, segundo cada procedimento observado associando às condutas da comunicação terapêutica que foram apresentados nas tabelas.

\section{Enfermeiro A}

O primeiro profissional (enfermeiro A), sexo feminino, 31 anos, 10 anos de atuação profissional. Trabalhava 40 horas/semana na Estratégia Saúde da Família e no hospital, assumindo em média cinco plantões/mês.

\section{- Visita aos Pacientes}

Iniciou a visita apresentando-se e a comunicação estabelecida direcionou-se a dados pontuais e únicos, no qual foram questionados: alimentação, eliminações, higiene e queixas álgicas. No exame físico, restringiu-se às auscultas pulmonar e cardíaca.

Toda visita de enfermagem deve ser baseada na entrevista e no exame físico. Além de sistematizada com início, meio e fim. O início (introdução) 
demarca-se pela apresentação do profissional, incluindo nome e função. Em seguida na abordagem do paciente pelo nome, antecedido dos corretos pronomes de tratamento e na explanação dos motivos da visita. O meio (desenvolvimento), ocasião nas quais as necessidades são identificadas, daí a importância de seguir roteiro norteador. Posteriormente, têm-se a execução do exame físico para pesquisar dados objetivos, complementando a investigação subjetiva. O fim (encerramento) é demarcado pela conscientização do paciente da finalização da visita, dando-Ihe a oportunidade para expor algo ainda não observado e primordial para o cuidar $^{(6)}$. Para tanto, a comunicação deve ser considerada, fator determinante para efetivação desse processo.

Diante do exposto, observa-se que a visita do enfermeiro A apresentou algumas falhas significativas, sobretudo nas duas últimas etapas (desenvolvimento e a finalização). O exame físico limitou-se às auscultas pulmonar e cardíaca e o histórico restringiu-se a poucos dados clínicos. O profissional não finalizou sua interação, deixando lacunas no processo comunicativo.

Durante a visita, o enfermeiro A, dentro da classificação de Stefanelli ${ }^{(4)}$, para o grupo de EXPRESSÃO, obteve os seguintes aspectos: VERBALIZOU INTERESSE, USOU FRASES INCOMPLETAS, REPETIU AS ÚLTIMAS PALAVRAS DITAS PELOS PACIENTES, FEZ PERGUNTAS E COLOCOU EM FOCO A IDÉIA PRINCIPAL. Quanto ao grupo de Validação, utilizou um item: REPETIR A MENSAGEM DO PACIENTE. O processo comunicativo pode ser caracterizado como terapêutico, já que os tópicos do grupo de expressão e de validação se sobressaem quantitativamente, como foi apresentado nas tabelas.

\section{- Sondagem Vesical}

A paciente havia se submetido à perineoplastia e cistopexia, encontrava-se no primeiro dia de pósoperatório e apresentava bexigoma, inquieta com queixas álgicas. $\mathrm{O}$ enfermeiro apresentou-se e comunicou a realização do procedimento. À medida que o executava, questionava desconforto e dores, e ao término, questionou a melhora à paciente. Apesar de haver diálogo, esse ocorreu de forma fria e praticamente unidirecional.

A interação entre enfermeiro e paciente deve ser personalizada, de modo que os pacientes percebam que os profissionais são mais do que indivíduos responsáveis pelo cuidado. Na execução de alguns cuidados/procedimentos, como a sondagem vesical, o diálogo, a troca de informações sobre termos e a explicação da sua execução, fundamentam uma interação terapêutica eficiente a fim de estabelecer uma relação de confiança ${ }^{(7)}$. Diante da sondagem vesical, pouco diálogo aconteceu e o processo comunicativo do enfermeiro A não foi eficiente, já que o procedimento em si não foi explicado e a troca de informações não foi estabelecida.

A comunicação é eficaz quando a mensagem transmitida torna-se comum. No entanto, esse processo pode ser afetado por vários fatores. Entre eles, sobressaem: inadequação do emissor na maneira de se expressar; falta de habilidade do emissor para transmitir a mensagem e do receptor de compreendê-la; alterações da mensagem durante a transmissão em virtude de falha no canal de comunicação ${ }^{(2,8)}$

O profissional, conforme a classificação de Stefanelli ${ }^{(4)}$ utilizou apenas quatro itens do grupo de expressão, são eles: VERBALIZAR INTERESSE, REPETIR COMENTÁRIOS FEITOS PELO PACIENTE, REPETIR AS ÚLTIMAS PALAVRAS DITAS PELO PACIENTE e FAZER PERGUNTAS. Quanto aos grupos de clarificação e validação, nenhum item foi utilizado. Mediante análise dos itens, a comunicação estabelecida pode ser considerada terapêutica.

\section{- Orientações sobre cirurgia}

O paciente encontrava-se no pré-operatório de apendicectomia, fácies triste e preocupado, sendo a primeira experiência frente ao procedimento cirúrgico. O profissional aproximou-se sem apresentação e iniciou a interação.

Muitos pacientes expressaram medo e apreensão frente à necessidade de cirurgia. Esses sentimentos são ainda mais freqüentes entre aqueles que serão submetidos pela primeira vez a um procedimento cirúrgico. Nesse sentido, a assistência de enfermagem engloba apoio emocional e psicológico $^{(9)}$.

Tal fato faz entender que a atuação do profissional A foi de extrema relevância para a situação em que o paciente se encontrava; visto que ofereceu suporte psicológico e de certa forma contribuiu para a tranqüilização do paciente.

O grupo de Expressão foi usado nos seguintes itens: USAR FRASES INCOMPLETAS, REPETIR AS ÚLTIMAS PALAVRAS DITAS PELO PACIENTE, FAZER PERGUNTAS RELATIVAS AOS DADOS COMUNICADOS. Os grupos de Clarificação e Validação não foram praticados. Diante do contexto, a comunicação estabelecida é caracterizada como não-terapêutica.

\section{- Verificação da Pressão Arterial (P.A)}

O enfermeiro apenas se aproximou da cliente, uma parturiente, e sem apresentar-se, colocou o esfigmomanômetro no braço, verificando o valor e saiu sem interação.

A verificação dos sinais vitais é uma rotina hospitalar que oferece possibilidades de interação entre a equipe de enfermagem e os pacientes, e, portanto, é uma oportunidade para estabelecer 
comunicação, sobretudo a verbal ${ }^{(10)}$. Nesse momento os pacientes devem ser informados dos valores obtidos e da sua representação.

Comunicação adequada é conversar e receber informações pertinentes aos interesses do paciente; é entender o que o outro quer transmitir e fazê-lo sentir-se bem atendido ${ }^{(4)}$.

O profissional A não se comunicou verbalmente com a parturiente. Apesar de ser um procedimento simples e rotineiro, envolve o paciente nesse processo. Devido a unidirecionalidade a comunicação foi classificada não-terapêutica.

\section{- Avaliação das Parturientes}

Ainda no setor obstétrico, o profissional A procedeu à avaliação de algumas parturientes que lá estavam, com ausculta dos batimentos cárdio-fetais (BCFs) e orientações para facilitar o parto. Nessa interação, o profissional não se apresentou e não seguiu seqüência lógica, essencial no contato interpessoal.

Existe a necessidade de comunicação entre enfermeiros e parturientes; porém, esta não ocorre ou possui muitas falhas às quais são causadas por ruídos comunicacionais ${ }^{(11)}$.

O processo comunicativo do enfermeiro $A$, caracterizou-se da seguinte forma: grupo de Expressão, dois itens (FAZER PERGUNTAS, FAZER PERGUNTAS RELATIVAS AOS DADOS COMUNICADOS) e Validação, um item (REPETIR A MENSAGEM DO PACIENTE). Observa-se, portanto, que a comunicação foi falha e não-terapêutica.

\section{Enfermeiro B}

O enfermeiro $B$, sexo masculino, 24 anos e em efetivo exercício há um ano. Trabalhava 40 horas/semana na Estratégia Saúde da Família, e no hospital em média três plantões/mês.

\section{- Visita aos Pacientes}

O profissional ao adentrar nas enfermarias não se apresentou e abordou os pacientes mediante dados contidos nos prontuários. Interrogou queixas e/ou alterações notificadas, não realizando exame físico. A visita de enfermagem diária é um importante artifício para identificar o nível da necessidade de segurança, amor, auto-estima, espiritualidade e biofisiolológica do paciente ${ }^{(12)}$. Nesse contexto, o enfermeiro apresentou uma comunicação falha e pouco sistemática, na qual as técnicas comunicativas necessitam preconizar interações com início, meio e fim $^{(6)}$ bem como o embasamento científico, foram negligenciados.

À caracterização de Stefanelli(4), o profissional usou quatro itens do grupo de Expressão: FAZER PERGUNTAS, MANTER O PACIENTE NO MESMO ASSUNTO, COLOCAR EM FOCO A IDÉIA PRINCIPAL $\mathrm{C}$ USAR FRASES DESCRITIVAS.
Usar frases descritivas, é uma técnica muito usada no relacionamento terapêutico, quando se explica ao paciente/família, rotinas da unidade, procedimentos e orientações. Deve-se ter cuidado em não confundir $o$ uso de frases descritivas e afirmativas com conselhos ${ }^{(4)}$.

O cuidar humanizado não se concretiza se estiver centralizado unicamente na doença, e os profissionais também devem dispor das devidas condições para desenvolver suas habilidades comunicacionais. Quando se percebe que o paciente também tem dificuldade em expressar-se de forma descritiva, deve-se valer de outras técnicas de comunicação para ajudá-lo a se expressar mais claramente.

Quanto à comunicação não-terapêutica, dois itens foram utilizados: MUDAR DE ASSUSTO INADEQUADAMENTE E "NÃO SABER OUVIR". Apesar de a comunicação terapêutica ter sido utilizada (dois itens), houve possibilidade de contemplação dos demais, no entanto, não foram praticados.

\section{- Realização do Curativo Cirúrgico I}

A segunda interação promovida pelo enfermeiro $B$, foi a realização de um curativo. Tratava-se de uma ferida cirúrgica, secundária à apendicectomia. Ao chegar à enfermaria, sem se apresentar, perguntou quem era "a pessoa do curativo".

$\mathrm{Na}$ perspectiva da comunicação terapêutica, o profissional usou apenas 2 itens do grupo de Expressão: FAZER PERGUNTAS e FAZER PERGUNTAS RELATIVAS AOS DADOS COMUNICADOS. Na comunicação não-terapêutica, USOU JARGÃO PROFSSIONAL e MUDOU DE ASSUNTO INADEQUADAMENTE.

A comunicação necessita sempre ser transmitida de maneira simples, clara e objetiva, sem o uso de termos difíceis, para a compreensão até de pessoas com menos escolaridade ${ }^{(13)}$.

Pode-se afirmar que os itens de Expressão são insuficientes para assegurar uma comunicação terapêutica de acordo com as estratégias de Stefanelli ${ }^{(4)}$, à medida que o uso de termos técnicos e a mudança de assunto inadequada ao contexto, somadas a negligência da sequência comunicativa, listada por Vasconcellos(10), como a aproximação do paciente, chamá-lo pelo nome, orientá-lo sobre o procedimento e esclarecer suas dúvidas; caracterizam a predominância da comunicação não terapêutica.

\section{- Realização do Curativo Cirúrgico II}

A terceira e última interação observada durante a atuação desse enfermeiro, foi a realização de outro curativo cirúrgico de ferida traumática na região inguinal esquerda. Quando se trata de regiões íntimas o profissional deve manter sempre a privacidade, prevalecendo a comunicação verbal nesse instante ${ }^{(6-7)}$. 
O enfermeiro B utilizou o grupo de Expressão PERMANECENDO EM SILÊNCIO. No tocante à comunicação não-terapêutica USOU TERMOS COM CONOTAÇÃO DE JULGAMENTO e JARGÃO PROFISISONAL.

Por não ser de domínio do cliente, este não compreende, devidamente, a linguagem adotada entre profissionais de saúde ${ }^{(14)}$. Desse modo, ele pode fazer interpretações errôneas dos cuidados prestados e das condições do seu estado, o que pode influenciar sobremaneira.

Somente esse último tópico referente ao grupo de expressão foi observado, não caracteriza a comunicação estabelecida aqui como terapêutica.

\section{Enfermeiro C}

O terceiro enfermeiro, C, sexo feminino, 37 anos, com 12 anos de atuação profissional. Trabalhava 40 horas/semana na Estratégia Saúde da Família e no hospital, em média cinco plantões/mês.

\section{- Visita aos Pacientes}

O profissional C identificou através dos prontuários quais os pacientes mais debilitados anotando o nome e o leito, além de informações contidas nos registros de enfermagem para prosseguir com a visita.

Ao adentrar as enfermarias o profissional apresentou-se, em seguida, abordou os pacientes pelo nome. Não realizou exame físico, pois segundo ele, é muito difícil examinar todos os pacientes integralmente, já que além da grande demanda de pessoas a serem atendidas, há ainda as responsabilidades burocráticas. Finalizou a visita com cada paciente, informando o nome do auxiliar e/ou técnico de enfermagem "responsável" por eles no plantão, esclarecendo dúvidas acerca das rotinas da instituição e se dispondo a ajudá-los. É a partir da visita de enfermagem que o enfermeiro estabelece um processo de comunicação ${ }^{(4,12)}$.

$O$ enfermeiro $C$ estabeleceu um processo comunicativo eficaz, já que seguiu uma seqüência, além de ter prestado assistência holística. Um estudo realizado em uma unidade básica mostrou que uma barreira da empatia no cuidado ministrado pelos enfermeiros, foi a falta de tempo de tempo em detrimento à grande demanda de cliente ${ }^{(15)}$.

Observa-se que o mesmo argumento identificado no estudo citado em relação a grande demanda de pacientes a serem atendidos, foi o mesmo atribuído pelo enfermeiro $C$ ao justificar a não realização do exame físico durante as visitas; conclui-se, portanto, que o quantitativo de pacientes e a burocracia existente interferem de forma negativa no processo comunicativo terapêutico.

No entanto, apesar das barreiras citadas, o profissional $\mathrm{C}$ conseguiu praticar segundo a classificação de Stefanelli ${ }^{(4)}, 12$ itens do grupo de
Expressão, a serem citados: PERMANECER EM SILÊNCIO, OUVIR REFLEXIVAMENTE, VERBALIZAR ACEITAÇÃO, VERBALIZAR INTERESSE, USAR FRASES INCOMPLETAS, REPETIR COMENTÁRIOS FEITOS PELOS PACIENTES, FAZER PERGUNTAS RELATIVAS AOD DADOS COMUNICADOS, USAR FRASES DESCRITIVAS, MANTER O PACIENTE NO MESMO ASSUNTO, COLOCAR EM FOCO A IDÉIA PRINCIPAL.

Quanto ao grupo de Clarificação, dois itens: SOLICITAR QUE ESCLAREÇA TERMOS INCOMUNS $\mathrm{E}$ DESCREVER OS EVENTOS EM SEQUÊNCIA LÓGICA; um item do grupo de Validação: SUMARIZAR O QUE FOI DITO NA I NTERAÇÃO.

A validação da comunicação deve acompanhar todo processo de relacionamento terapêutico e é necessária porque mensagens emitidas devem ter a mesma significação para as pessoas envolvidas no processo terapêutico ${ }^{(4)}$. A validação da mensagem deve ser feita tão logo surja sua necessidade. Ainda segundo as concepções da teórica, se as mensagens do paciente não forem clarificadas e validadas, incorre-se o risco de agir com base em valores próprios, crenças ou interpretações errôneas.

Chama atenção o fato, de mesmo com as dificuldades citadas para o estabelecimento da comunicação eficaz, o enfermeiro $C$, diante da caracterização mostrada, conseguiu se comunicar terapeuticamente dentro dos três grupos.

\section{- Orientações referente a procedimento cirúrgico}

A segunda interação observada diz respeito às orientações ao paciente sobre cirurgia. $O$ paciente encontrava-se no pré-operatório de apendicectomia, ansioso e introspectivo. O profissional aproximou-se oferecendo orientações minuciosas sobre o processo cirúrgico e cuidados pós-operatórios.

Muitos pacientes expressam medo e apreensão frente à necessidade da cirurgia. Esses sentimentos são ainda mais freqüentes entre aqueles indivíduos que se submetem pela primeira vez a um procedimento cirúrgico. Daí a necessidade do enfermeiro estar sempre atento para tranquilizá-lo nesse período ${ }^{(9)}$.

O enfermeiro também tocou afetivamente o paciente, sendo o primeiro e único profissional observado a realizá-lo. A comunicação não-verbal na enfermagem muitas vezes se limita a realização dos procedimentos, porém 0 enfermeiro deve estar consciente que o paciente apresenta necessidade de ser tocado ${ }^{(4,6,16)}$. Assim sendo, o toque foi essencial para minimizar a apreensão do paciente e melhorar seu estado emocional. $O$ enfermeiro contribuiu para uma assistência holística já que os sentimentos do paciente foram considerados e as suas necessidades identificadas.

No que se refere às estratégias de comunicação, o enfermeiro $C$ utilizou oito tópicos do grupo de 
Expressão: PERMANECER EM SILÊNCIO, OUVIR REFLEXIVAMENTE, USAR FRASES INCOMPLETAS, REPETIR AS ÚLTIMAS PALAVRAS DITAS PELO PACIENTE, FAZER PERGUNTAS, FAZER PERGUNTAS RELATIVAS AOS DADOS COMUNICADOS, MANTER O PACIENTE NO MESMO ASSUNTO, COLOCAR EM FOCO A IDÉlA PRINCIPAL. Utilizou 1 tópico do grupo de Clarificação: DESCREVER OS EVENTOS EM SEQÜÊNCIA LÓGICA e 1 item do grupo de Validação: SUMARIZAR O QUE FOI DITO NA INTERAÇÃO. De forma clara esse enfermeiro comunicou-se terapeuticamente.

\section{- Verificação da PA e da Temperatura}

A verificação da P.A e da temperatura comportase como a terceira interação estabelecida pelo enfermeiro C. O profissional ao aproximar-se da paciente, se apresentou e deixou claro que estava ali para ajudá-la; ao término, informou os valores obtidos na verificação.

$O$ enfermeiro $C$ procedeu de forma correta ao verificar a P.A e temperatura da parturiente, apesar de serem considerados como procedimentos simples e de rotina. Em relação ao processo comunicativo, agiu certo ao se apresentar e principalmente ao informar os valores aferidos à paciente. Quando esse é informado sobre seu estado de saúde de forma clara e objetiva, na maioria das vezes se sente melhor e atua como um partícipe na sua recuperação ${ }^{(6,17)}$.

Durante a interação, o enfermeiro $C$ usou três itens de Expressão: PERMANECER EM SILÊNCIO, VERBALIZAR INTERESSE e USAR FRASES DESCRITIVAS.

A clarificação torna-se necessária quando o paciente não se expressa com clareza, não consegue compreender com precisão a experiência que vivencia, seus pensamentos e sentimentos e quando não descreve os eventos em seqüência lógica. Ao clarificar o conteúdo expresso pelo paciente o enfermeiro oferece-lhe novas oportunidades de correção da informação. $O$ foco desse grupo é obter um significado mais explícito da mensagem. Quando estas técnicas são usadas com interesse genuíno, elas são percebidas pelo paciente como sinal de interesse em compreendê-lo( ${ }^{(4)}$.

Apesar dos itens de Clarificação e Validação não terem sido utilizados, o processo comunicativo ainda pode ser caracterizado como terapêutico.

\section{- Toque Vaginal}

A quarta interação observada foi o toque vaginal de uma parturiente nulípara. $O$ enfermeiro se apresentou e explicou o procedimento à mulher. Ao ser explicado o procedimento, a parturiente demonstrou certa rejeição, porém permitiu que o profissional realizasse-o. Ao observar as reações dos pacientes quando sentem sua privacidade ameaçada, ou mesmo invadida, pode-se identificar sentimentos como constrangimento, vergonha, embaraço e revolta. Por outro lado, demonstram satisfação e agradecimento através de expressões corporais ou verbais ao sentirem-se mais seguros quando se colocam biombos para execução de procedimentos ${ }^{(7)}$.

A princípio, a parturiente sentiu sua privacidade ameaçada, ainda quando o profissional explicava o procedimento. No entanto, a comunicação verbal promovida pelo enfermeiro fez com que a paciente reduzisse esse sentimento e permitindo a execução do toque. É importante salientar que a parturiente estava na maternidade e compartilhava o setor com mulheres que se encontravam na sua mesma situação. Isso contribui para uma maior segurança da paciente, bem como com as demais e para um sentimento de privacidade preservado, já que todas dividem as mesmas angústias e dificuldades.

Durante o toque vaginal, o enfermeiro $C$ usou quatro itens do grupo de Expressão: VERBALIZAR INTERESSE, FAZER PERGUNTAS, USAR FRASES DESCRITIVAS, COLOCAR EM FOCO A IDÉIA PRINCIPAL. No que se refere ao grupo de Clarificação, O DESCREVER OS EVENTOS EM SEQUÊNCIA LÓGICA. O enfermeiro foi unidirecional em muitos momentos. Mesmo assim, sobressaiu os itens terapêuticos.

\section{- Acompanhamento da Evolução e do Trabalho de Parto Vaginal}

O acompanhamento da evolução e do trabalho de parto normal foi a quinta interação realizada pelo profissional C. A multípara, quinta gestação. Logo no início de sua internação evoluiu rapidamente para o trabalho de parto. O profissional apresentou-se e esteve com a mulher de forma integral, até o delivramento total da placenta. Durante o contato, deixou claro a relação de ajuda para atender suas necessidades.

O preparo da mulher durante a gestação e a assistência no momento do parto, oferece a mesma possibilidade de vivenciar a experiência parto como processo fisiológico. O enfermeiro desempenha um papel relevante de facilitador. Assim, articulando seu conhecimento técnico/científico com as estratégias comunicativas, contribui para que o parto seja mais ameno e menos sofrível para a mulher ${ }^{(11)}$.

Constata-se que o enfermeiro $C$, ofereceu assistência eficaz à mulher, já que, ao realizar o procedimento, a estimulando para interagir com o profissional e com o meio. O enfermeiro utilizou cinco itens do grupo de Expressão: OUVIR REFLEXIVAMENTE, VERBALIZAR ACEITAÇÃO, VERBALIZAR INTERESSE, DEVOLVER A EXPRESSÃO FEITA PELO PACIENTE $P$ USAR FRASES DESCRITIVAS. Utilizou um item do grupo de Clarificação: DESCREVER OS EVENTOS EM SEQÜÊNCIA LÓGICA. Apesar do grupo de Validação 
não ter sido utilizado em nenhum tópico, a comunicação estabelecida nesta interação pode ser considerada como terapêutica.

\section{- Orientações à Parturiente}

As orientações à parturiente correspondem à sexta interação promovida pelo enfermeiro C. O profissional apresentou-se e estimulou a parturiente à deambulação e ao uso do "cavalinho", como forma de acelerar a evolução do trabalho de parto.

A gestante, sobretudo as primíparas, devem ser preparadas para o trabalho de parto, como forma de orientação e suporte a esse processo(2). O enfermeiro teve uma importante atuação, com a comunicação verbal estimulou a mulher a se tornar mais ativa.

O enfermeiro $C$ utilizou seis itens do grupo de Expressão: PERMANECER EM SILÊNCIO, OUVIR REFLEXIVAMENTE, VERBALIZAR INTERESSE, FAZER PERGUNTAS, FAZER PERGUNTAS RELATIVAS AOS DADOS COMUNICADOS U USAR FRASES DESCRITIVAS. Um item do grupo de Clarificação: DESCREVER OS EVENTOS EM SEQÜÊNCIA LÓGICA e um tópico de Validação: REPETIR A MENSAGEM DO PACIENTE. As estratégias não-terapêuticas estiveram ausentes durante toda interação, caracterizando o processo comunicativo como predominantemente terapêutico.

\section{- Orientações às Puérperas}

O profissional C realizou como sétima interação, as orientações às puérperas na unidade de alojamento conjunto. O enfermeiro se apresentou, perguntou quais as principais dúvidas dessas mulheres e as orientou quanto à amamentação, à higiene, à vacinação dos recém-nascidos.

Avaliar o conhecimento das mulheres que estão no puerpério, no que se refere à amamentação, consulta puerperal, higiene, entre outros, repercutirá na qualidade dos serviços e no bem-estar do binômio mãe-filho. O conhecimento materno é considerado como responsabilidade primordial da enfermagem ${ }^{(2)}$. A atuação desse profissional se adequai ao preconizado pela referência acima citada, já que as orientações foram promovidas e as mulheres devidamente avaliadas sob os aspectos citados.

O enfermeiro $C$ utilizou cinco itens do grupo de Expressão: PERMANECER EM SILÊNCIO, OUVIR REFLEXIVAMENTE, VERBALIZAR INTERESSE, FAZER PERGUNTAS, USAR FRASES DESCRITIVAS. UsOu Um item de Validação: SUMARIZAR O QUE FOI DITO NA INTERAÇÃO, e um item para a comunicação nãoterapêutica: DAR CONSELHOS. O item referente à comunicação não-terapêutica foi utilizado principalmente nas orientações sobre amamentação, quando algumas mães se recusavam a praticá-la. Em todo caso, há a prevalência dos itens terapêuticos; caracterizando desse modo a comunicação estabelecida na interação.

\section{Orientações à parturiente I I}

A oitava interação promovida pelo enfermeiro C, direciona-se às orientações à paciente que se submeteria à cesariana. O profissional ao interagir com ela, apresentou-se e procurou ouvi-la identificando quais os problemas de enfermagem. Mediante o encontrado, orientou, e tocou a paciente. Atenta-se ao fato, de como o toque nessa interação foi terapêutico, a mulher demonstrou mais confiança no profissional.

As situações ocorridas no processo comunicacional diferem umas das outras e para decifrá-las, somos obrigados a paralisar sua dinâmica, a fim de podermos analisá-la como um todo, devido à sua complexidade e multidimensionalidade ${ }^{(11)}$. Evidencia-se que o enfermeiro $C$ praticou o processo comunicativo de forma eficaz, pois primeiro identificou a apreensão e angústia da mulher, em seguida pesquisou quais fatores inter-relacionados. Assim, ao intervir de maneira direta, estabeleceu um vínculo terapêutico ainda mais ratificado com o toque. O toque usado ofereceu premissas para a prática do cuidar humanístico.

Para a classificação de Stefanelli(4), mais uma vez, o enfermeiro $C$ usou com predominou a comunicação terapêutica, utilizando 14 itens do grupo de Expressão: PERMANECER EM SILÊNCIO, OUVIR REFLEXIVAMENTE, VERBALIZAR ACEITAÇÃO, VERBALIZAR INTERESSE, USAR FRASES INCOMPLETAS, REPETIR COMENTÁRIOS FEITOS PELO PACIENTE, FAZER PERGUNTAS, FAZER PERGUNTAS RELATIVAS AOS DADOS COMUNICADOS, INTRODUZIR PROBLEMA RELACIONADO, DEVOLVER A EXPRESSÃO FEITA PELO PACIENTE, USAR FRASES DESCRITIVAS, DIZER “NÃO" e ESTIMULAR EXPRESSÃO DE SENTIMENTOS SUBJACENTES. Usou ainda um item do grupo de Validação: REPETIR A MENSAGEM DO PACIENTE.

\section{- Punção Venosa}

A punção venosa representa a nona e última interação observada durante o plantão do enfermeiro C. O paciente, idoso (80 anos), estava na clínica médica, acometido por Acidente Vascular Cerebral, inconsciente, porém responsivo aos estímulos dolorosos, com paralisia e parestesia nos membros. Segundo o prontuário, há uma semana apresentava episódios diarréicos e desidratação, fato dificultador para punção venosa agravado pela incontinência urinária. O enfermeiro se apresentou e mesmo percebendo a apatia e inconsciência do paciente, explicou o procedimento.

A comunicação terapêutica deve ser praticada com todos os doentes, até mesmo com as pessoas inconscientes, já que essa situação não reduz o paciente enquanto ser humano e este independente do quadro precisa conhecer o procedimento a que 
será submetido a merece ser informado acerca o ambiente que o envolve ${ }^{(1,18)}$. Apesar de nenhuma resposta verbal do paciente, o profissional se comunicou, explicando o procedimento e se relacionando com o "cliente" durante toda interação.

Utilizou três itens para o grupo de Expressão: PERMANECER EM SILÊNCIO, VRBALIZAR INTERESSE, USAR FRASES DESCRITIVAS. Referente à comunicação não-terapêutica, apenas ofereceu falsa tranqüilização. Verifica-se que o enfermeiro $C$ agiu terapeuticamente com o paciente.

\section{Enfermeiro D}

O enfermeiro D, sexo masculino, 26 anos, cinco anos de atuação profissional. Atua integralmente no ambiente hospitalar; na instituição onde a pesquisa foi desenvolvida, assume em média dois plantões/mês.

\section{- Punção Venosa}

Ao adentrar a enfermaria, do mesmo paciente relatado acima, cumprimentou a profissional $\mathrm{C}$ e perguntou se o doente estava inconsciente. Depois disso, a comunicação praticada pelo enfermeiro $D$, direcionou-se exclusivamente ao seu colega de profissão.

A inconsciência é uma condição em que a função cerebral está deprimida, variando do torpor ao coma. Nesse sentido, a comunicação proporcionada e a eficácia do cuidado de enfermagem oferecido ao paciente inconsciente podem significar a diferença entre a vida e a morte ${ }^{(6)}$. Observa-se ao analisar o comportamento do enfermeiro $D$ durante a punção venosa, visto que nenhuma ação verbal foi direcionada ao paciente.

Para a classificação de Stefanelli(4) o enfermeiro utilizou um item do grupo de Expressão: PERMANECER EM SILÊNCIO e 2 tópicos referentes à classificação não-terapêutica: COMUNICAÇÃO UNI DIRECIONAL e USAR J ARGÃO PROFISSIONAL.

Por deter os conhecimentos científicos, o enfermeiro necessita compreender que a troca de papéis no ato comunicativo existe e é constante. Caso contrário forçará um unidirecionamento da mensagem, sem a influência do comportamento do paciente durante a interação ${ }^{(4,17)}$.

O processo comunicativo durante a interação do enfermeiro D com o paciente foi, portanto, nãoterapêutica.

\section{Enfermeiro E}

Já o enfermeiro $E$, sexo feminino, 56 anos, 22 anos de profissão. Atua apenas no ambiente hospitalar especificamente na instituição assumindo em média três plantões/mês.

\section{I munização das Puérperas}

A imunização das três puérperas internadas foi a primeira interação estabelecida pelo enfermeiro $E$, no qual entrou na enfermaria, não se apresentou e foi direto preparar as vacinas. Ao administrar, explicou a finalidade da imunização.

A comunicação numa relação terapêutica deve ser praticada em toda e qualquer interação ${ }^{(6,12)}$.

Dentro do grupo de Expressão, utilizou dois itens: PERMANECER EM SILÊNCIO e USAR FRASES DESCRITIVAS. Para a comunicação não-terapêutica três tópicos foram utilizados: COMUNICAÇÃO UNIDIRECIONAL, USAR JARGÃO PROFISSIONAL $\mathrm{E}$ MUDAR DE ASSUNTO INADEQUADAMENTE. A comunicação estabelecida pelo enfermeiro $E$ durante a imunização das puérperas foi portanto, nãoterapêutica.

\section{- Sondagem Vesical}

A sondagem vesical foi a segunda interação realizada pelo enfermeiro. A paciente estava no segundo dia de pós-operatório de perineoplastia e cistopexia, apresentava bexigoma e queixas álgicas. O profissional não se apresentou; perguntou sobre seu estado geral e lhe informou da sondagem.

A comunicação é necessária também durante a sondagem vesical, já que é um procedimento invasivo $^{(7,12)}$ e desconhecido ainda por muitos. Nesse contexto, a comunicação estabelecida pelo profissional não foi eficaz e o vínculo não estabelecido.

Durante a interação, o profissional C utilizou três itens do grupo de Expressão: FAZER PERGUNTAS, FAZER PERGUNTAS RELATIVAS AOS DADOS COMUNICADOS e USAR FRASES DESCRITIVAS e 3 itens para a comunicação não-terapêutica: OFERECER FALSA TRANQUI LIZAÇÃO

COMUNICAÇÃO UNIDIRECIONAL E USAR JARGÃO PROFISSIONAL. Como o quantitativo de itens se equiparou, caracteriza-se o processo comunicativo como não-terapêutico, já que a paciente não foi devidamente informada sobre o procedimento, e isto é premissa básica de toda interação.

\section{- Realização de Curativo Contaminado}

Tratava-se de queimaduras de $2^{\circ}$ e $3^{\circ}$ graus nos membros inferiores. O profissional ao entrar na enfermaria, questionou o agente causador e enquanto realizava o curativo, tentava confortar a paciente.

Observa-se que apesar do enfermeiro não ter se apresentado, preocupou-se com o estado emocional da paciente, através da comunicação verbal, tentou confortá-la e fazer com que se adaptasse em relação à situação. É importante compreender que o paciente é um ser humano e que vários aspectos além do clínico o envolvem. Desse modo, a cultura, o ambiente, os aspectos socioeconômicos, devem ser 
considerados, já que o CUIDAR efetivo, manifesta-se mediante a valorização desses fatores ${ }^{(13,17)}$.

Quanto à comunicação terapêutica, utilizou quatro itens do grupo de Expressão: PERMANECER EM SILÊNCIO, VERBALIZAR INTERESSE, FAZER PERGUNTAS, FAZER PERGUNTAS RELATIVAS AOS DADOS COMUNICADOS e 2 itens referentes à comunicação não-terapêutica: DAR RESPOSTA ESTEREOTIPADA, USAR JARGÃO PROFISSIONAL. Considerando principalmente a melhora do estado emocional da paciente, após a interação, pode-se caracterizar a comunicação estabelecida, como terapêutica.

Porém, um fato desse ser analisado com minúcia, esse profissional foi o que mais usou o jargão profissional nas três interações que efetuou. A linguagem comum favorece a efetivação da mensagem que a enfermeira pretende transmitir ao paciente, corroborando com as ações com vistas a preservação da saúde e tratamento de doenças.

\section{CONCLUSÕES}

Constatou-se que, embora os enfermeiros não se atentem na maioria das vezes para as técnicas comunicativas, até mesmo por não conhecê-las, as utilizam cotidianamente. Por não darem a devida importância à comunicação como um processo, se comunicam de forma pouco eficaz e não direcionada.

De acordo com os dados apresentados e discutidos, constata-se que os itens não-terapêuticos estiveram presentes nas interações da maioria dos enfermeiros, principalmente, o uso de linguagem técnica. Apesar do hábito adquirido ainda na academia de utilizar termos técnicos ao se comunicarem, não devem se fazer presentes já que se comportam como barreiras comunicativas.

De modo a tornar a comunicação terapêutica presente nas interações no cotidiano das instituições hospitalares, sugerem-se discussões sobre dimensionamento de profissionais de enfermagem, pois as situações observadas sinalizam que não é possível se realizar uma assistência integral, o cuidado fica comprometido e, consequentemente, o processo comunicativo torna-se falho.

Outro ponto que se destaca com a realização do presente estudo é a importância de investimento na educação permanente, a qual deveria ser meta de gestores que poderiam eleger o tema para se trabalhar no próprio local de trabalho como forma de alertar e esclarecer os profissionais (não apenas os enfermeiros) sobre a importância da comunicação terapêutica. Ressalta-se também a importância do ensino comunicativo na graduação, visto que matriz curricular da maioria dos cursos de enfermagem ainda não aborda a comunicação. Acredita-se que essa habilidade possa ser adquirida pelos profissionais de saúde com vista a melhoria da assistência.

\section{REFERÊNCI AS}

1. Teixeira AL, Braga A, Esteves MC. A comunicação com a criança na punção venosa: percepção do enfermeiro. Revista do Hospital de Crianças Maria Pia. 2004; 13(3): 187-94.

2. Morais GSN, Costa SFG, Fontes WD, Carneiro AD. Comunicação como instrumento básico no cuidar humanizado em enfermagem ao paciente hospitalizado. Acta paul. enferm. 2009; 22(3):54-63.

3. Machado MMT, Leitão GCM, Holanda FUX. O conceito de ação comunicativa: uma contribuição para a consulta de enfermagem. Rev Lat Am Enfermagem. 2005; 13(5): 723-28.

4. Stefanelli MC. Comunicação com paciente: Teoria e ensino. 2a ed. São Paulo: Robe; 1993.

5. Marcondi MA, Lakatos EM. Metodologia do Trabalho Científico. 6.ed. São Paulo: Atlas; 2006.

6. Potter PA, Perry AG. Fundamentos de enfermagem. 8.ed. Rio de Janeiro: Guanabara Koogan; 2005.

7. Pupulim JSL, Sawada NO. Reflexões acerca as comunicação enfermeiro-paciente relacionada à invasão da privacidade. Proceedings of 8Simpósio Brasileiro de Comunicação em Enfermagem 2002; São Paulo: Brasil; 2002.

8. Noreña-Peña AL, Cibanal-Juan L. El contexto de la interacción comunicativa: factores que influyen en la comunicación entre los profesionales de enfermería y los niños hospitalizados. Cultura de los cuidados. 2008; 23(1): 70-9.

9. Vargas TVP, Maia EM, Dantas RAS. Sentimentos de pacientes no pré-operatório de cirurgia cardíaca. Rev Lat Am Enfermagem. 2006; 14(3):383-88.

10. Puggina ACG, Silva MJP. Vital signs and facial expression of patients in coma. Rev Bras Enferm. 2009; 62(3): 435-41.

11. Almeida Cal, Tanaka OY. Perspectiva das mulheres na avaliação do Programa de Humanização do Pré-Natal e Nascimento. Rev Saude Publica. 2009; 43(1): 98-104.

12. Oriá MOB, Moraes LMP, Victor JF. A comunicação como instrumento do enfermeiro para o cuidado emocional do cliente hospitalizado. Rev. Eletr. Enf. [Internet] 2004 [cited 2010 mar 15];6(2):292-97. Available from: http://www.fen.ufg.br/revista/revista6_2/pdf/R4_co munica.pdf

13. Inaba LC, Silva MJP, Telles SCR. Paciente crítico e comunicação: visão de familiares sobre sua adequação pela equipe de enfermagem. Rev Esc Enferm USP. 2005; 39(4): 423-9.

14. Cavalcanti ACD, Coelho MJ. A linguagem como ferramenta do cuidado do enfermeiro em cirurgia cardíaca. Esc. Anna Nery. 2007; 11(2):220-6.

15. Takaki MH, Sant'ana DM. A empatia como essência no cuidado prestado ao cliente pela equipe de enfermagem de uma unidade básica de saúde. Cogitare Enferm. 2004; 9(1): 79-83. 
16. Oliveira OS, Nóbrega MML, Silva AT, Filha MOF. Comunicação terapêutica em enfermagem, revelada nos depoimentos de pacientes internados em centro de terapia intensiva. Rev. Eletr. Enf. [Internet] 2005 [cited 2010 mar 15];7(1):54-63. Available from: http://www. fen.ufg. br/revista/revista 7_1/original_05 htm.

17. Macêdo KNF, Pagliuca LMF. Características da comunicação interpessoal entre profissionais de saúde e deficientes visuais. Rev Paul Enferm. 2005; 23(3):221-26.

18.López ML, Carvalho EC. La comunicación terapêutica durante instalación de venoclisis: uso de la simulación filmada. Rev Lat Am Enfermagem. 2006; 14(5): 658-65.

Artigo recebido em 15.12.08.

Aprovado para publicação em 23.11.09.

Artigo publicado em 31.03.10. 